\title{
HYPERVELOCITY STARS AND THE RESTRICTED PARABOLIC THREE-BODY PROBLEM
}

\author{
Re'em Sari ${ }^{1,2}$, Shiho Kobayashi ${ }^{1,3}$, and Elena M. Rossi ${ }^{1}$ \\ ${ }^{1}$ Racah Institute of Physics, Hebrew University, Jerusalem 91904, Israel \\ 2 Theoretical Astrophysics 350-17, California Institute of Technology, Pasadena, CA 91125, USA \\ ${ }^{3}$ Astrophysics Research Institute, Liverpool John Moores University, Birkenhead CH41 1LD, UK \\ Received 2009 October 13; accepted 2009 November 16; published 2009 December 14
}

\begin{abstract}
Motivated by detections of hypervelocity stars that may originate from the Galactic center, we revisit the problem of a binary disruption by a passage near a much more massive point mass. The six orders of magnitude mass ratio between the Galactic center black hole $(\mathrm{BH})$ and the binary stars allows us to formulate the problem in the restricted parabolic three-body approximation. In this framework, results can be simply rescaled in terms of binary masses, their initial separation, and the binary-to-black hole mass ratio. Consequently, an advantage over the full three-body calculation is that a much smaller set of simulations is needed to explore the relevant parameter space. Contrary to previous claims, we show that, upon binary disruption, the lighter star does not remain preferentially bound to the black hole. In fact, it is ejected in exactly $50 \%$ of the cases. Nonetheless, lighter objects have higher ejection velocities, since the energy distribution is independent of mass. Focusing on the planar case, we provide the probability distributions for disruption of circular binaries and for the ejection energy. We show that even binaries that penetrate deeply into the tidal sphere of the $\mathrm{BH}$ are not doomed to disruption, but survive in $20 \%$ of the cases. Nor do these deep encounters produce the highest ejection energies, which are instead obtained for binaries arriving to $0.1-0.5$ of the tidal radius in a prograde orbit. Interestingly, such deep-reaching binaries separate widely after penetrating the tidal radius, but always approach each other again on their way out from the BH. Finally, our analytic method allows us to account for a finite size of the stars and recast the ejection energy in terms of a minimal possible separation. We find that, for a given minimal separation, the ejection energy is relatively insensitive to the initial binary separation.
\end{abstract}

Key words: binaries: general - Galaxy: center - Galaxy: halo - Galaxy: kinematics and dynamics - Galaxy: stellar content

Online-only material: color figure

\section{INTRODUCTION}

Hypervelocity stars (HVSs) are stars with a velocity exceeding the escape velocity of the Galaxy. Currently, 16 such stars have been observed, 15 of which are thought to originate from our Galactic center (Brown et al. 2005, 2006, 2007, 2009; Hirsch et al. 2005) and one from the Large Magellanic Cloud (Edelmann et al. 2005). All have been observed with radial velocities between $300 \mathrm{~km} \mathrm{~s}^{-1}$ and $800 \mathrm{~km} \mathrm{~s}^{-1}$ and almost all are located over $50 \mathrm{kpc}$ away. For stars whose likely origin is within the Galaxy and taking the Galactic potential into account, this translates to velocities of over $1000 \mathrm{~km} \mathrm{~s}^{-1}$ from the bulge. The observational strategy is such that most of the HVSs discovered are faint B stars. They have escape velocities from the surface of the order of $600 \mathrm{~km} \mathrm{~s}^{-1}$, well below the ejection velocity of $1000 \mathrm{~km} \mathrm{~s}^{-1}$. Thus, the standard mechanisms for producing high-velocity runaway stars, such as star scattering and explosion as a supernova of one component of a binary, cannot work. A dynamical interaction with a massive compact object is likely involved.

In this paper, we adopt one of the leading models for the formation of HVSs: the breakup of a binary as it approaches the BH in the Galactic center (Hills 1988). Simple analytical arguments can be made to show the potential of this model to explain HVSs.

If the binary of total mass $m$ has separation $a$, then tidal forces from the $\mathrm{BH}$ overcome the binary's mutual gravitational forces at the tidal radius $r_{t}=a(M / m)^{1 / 3}$, where $M$ is the mass of the $\mathrm{BH}$. The relative velocity of the binary components is of order $v_{0}=(G m / a)^{1 / 2}$. If the binary approaches the $\mathrm{BH}$ with negligible energy, its center of mass moves at the tidal radius with velocity of order $v_{\mathrm{BH}}=\left(G M / r_{t}\right)^{1 / 2}=v_{0}(M / m)^{1 / 3}$ relative to the $\mathrm{BH}$. There are three ways to estimate the energies of the individual components of the binary, assuming that they arrived with negligible total energy. It is instructive to consider all three:

1. Kinetic energy: adding or subtracting the relative velocity of the components, $v_{0}$, to the velocity around the BH results in an additional kinetic energy of order $v_{0} v_{\mathrm{BH}} \sim v_{0}^{2}(M / m)^{1 / 3}$.

2. Gravitational potential energy: the displacement of order $a$ in the position of each component of the binary, at a distance of about $r_{t}$ from the $\mathrm{BH}$, results in a change in gravitational energy of $G M a / r_{t}^{2} \sim v_{0}^{2}(M / m)^{1 / 3}$.

3. Work: the energy of each of the components in the $\mathrm{BH}$ frame is changing only due to mutual forces between the binary components. The force is of order $G m / a^{2}$ and the length, in the BH frame, over which it acts is $r_{t}$. Therefore, the work is $G m r_{t} / a^{2} \sim v_{0}^{2}(M / m)^{1 / 3}$.

All these estimates provide an energy of order $v_{0}^{2}(M / m)^{1 / 3}$. If the binary dissolves, one component of the binary stays bound to the $\mathrm{BH}$ and the other escapes with a velocity at infinity ${ }^{4}$ of $v_{0}(M / m)^{1 / 6}$. The encounter with the $\mathrm{BH}$, therefore, allows for a larger velocity by a factor of $(M / m)^{1 / 6}$ than the orbital velocity of the binary. For the parameters of our Galaxy and stars, $(M / m)^{1 / 6} \sim 10$, allowing ejections with velocities of thousands of kilometers per second.

\footnotetext{
4 We ignore the Galactic potential in this paper. By "the velocity at infinity" we mean the velocity of the object once it escapes the gravitational potential of the $\mathrm{BH}$ but has not yet climbed out of the potential of the rest of the Galaxy.
} 
This is the theoretical framework in which Hills (1988) predicted the existence of HVSs. It was later discussed by Yu \& Tremaine (2003) and Gould \& Quillen (2003). After the observational discovery, many more papers on the subject appeared (Gualandris et al. 2005; Ginsburg \& Loeb 2006a, 2006b; Bromley et al. 2006; Sesana et al. 2007; Perets et al. 2007; Kenyon et al. 2008; Tutukov \& Fedorova 2009), aiming to predict the properties of the ejected and/or captured stars. Other papers (e.g., Ginsburg \& Loeb 2007; Antonini et al. 2009) focused instead on the fate of binaries that are not dissolved, but that in fact coalesce. The investigations so far have used three-body simulations or analytic methods that relied on results from three-body simulations. Consequently, only a limited set of parameters (e.g., for the binary mass ratio) have been explored.

In this paper, we show that this problem can be investigated with methods related to those used in the study of asteroids in the solar system and which exploit the enormous disparity in mass between the bodies involved. Specifically, we can formulate it in terms of a restricted three-body problem, i.e., the motion of a single massless particle under the influence of external time-dependent forces. Our treatment is valid as long as the binary components are closer to each other than to the BH. Since $r_{t}=a(M / m)^{1 / 3}$ our approximation requires $(M / m)^{1 / 3} \gg 1$, which is fully satisfied in the case of the $\mathrm{BH}$ at the Galactic center and a binary of $\mathrm{B}$ stars, $(M / m)^{1 / 3} \cong 100$. With the advantages of this analytic method, we can reach general conclusions that do not depend on the physical properties of the system such as masses and the binary semimajor axis. Moreover, the orbit integration is faster and more stable, allowing us to handle more easily cases of close encounters between the bodies.

In Section 2, we outline the formulation of the three-body problem in terms of a restricted parabolic problem. In Section 3, we use the restricted radial problem to describe binaries that penetrate deep into the tidal radius. The radial problem has a singularity at the time that the binary encounters the $\mathrm{BH}$, and we use the results of the parabolic problem to pass the singularity and continue to describe the evolution of the binary on its trajectory away from the BH. In Section 4, we compare our results with three-body numerical integrations and find excellent agreement. We then use the parabolic and radial formalisms to investigate the probability of dissolving a circular, planar binary and to obtain quantitative estimates of the ejection velocities. We outline these results in Section 5.

\section{THE PARABOLIC RESTRICTED THREE-BODY PROBLEM}

\subsection{The Orbit}

In the case of interest $M / m \gg 1$ and the equation of motion for each of the binary members reads

$$
\begin{gathered}
\ddot{\mathbf{r}}_{1}=-\frac{G M}{r_{1}^{3}} \mathbf{r}_{1}+\frac{G m_{2}}{\left|\mathbf{r}_{1}-\mathbf{r}_{2}\right|^{3}}\left(\mathbf{r}_{2}-\mathbf{r}_{1}\right) \\
\ddot{\mathbf{r}}_{2}=-\frac{G M}{r_{2}^{3}} \mathbf{r}_{2}-\frac{G m_{1}}{\left|\mathbf{r}_{1}-\mathbf{r}_{2}\right|^{3}}\left(\mathbf{r}_{2}-\mathbf{r}_{1}\right),
\end{gathered}
$$

where $\mathbf{r}_{1}$ and $\mathbf{r}_{2}$ are the respective distances from the $\mathrm{BH}$. We therefore obtain that the equation for the distance between the two, $\tilde{\mathbf{r}} \equiv \mathbf{r}_{2}-\mathbf{r}_{1}$, is given by

$$
\ddot{\tilde{\mathbf{r}}}=-\frac{G M}{r_{2}^{3}} \mathbf{r}_{2}+\frac{G M}{r_{1}^{3}} \mathbf{r}_{1}-\frac{G m}{\tilde{r}^{3}} \tilde{\mathbf{r}} .
$$

Now, we assume that the two masses are much closer to each other, and to some effective parabolic trajectory $\mathbf{r}_{\mathrm{m}}$, than each of them is to the central mass $M$. As we already noticed in our introduction, this condition is actually enforced, up to the tidal radius, by the requirement of a large mass ratio $M / m \gg 1$. Deep inside the tidal sphere, this assumption may break and we will discuss the relevant conditions in Section 3.2.

We define the effective trajectory, $\mathbf{r}_{\mathrm{m}}$, to be the parabolic orbit set by the position and velocity of the center of mass of the binary when it is far away from the BH. The trajectory $r_{\mathrm{m}}$ is therefore described by

$$
r_{\mathrm{m}}=\frac{2 r_{p}}{1+\cos f},
$$

where $r_{p}$ is the distance of closest approach and $f$ the angle from the point of closest approach. The angle $f$, known as the true anomaly, is a function of time, but analytically one has only the time as a function of $f$ :

$$
\tilde{t}=\frac{\sqrt{2}}{3} \sqrt{\frac{r_{p}^{3}}{G M}} \tan (f / 2)\left(3+\tan ^{2}(f / 2)\right) .
$$

Note that the actual center of mass will not generally move on this orbit, since the total force on the binary does not equal the force that would act if the binary was a single body.

Linearizing the first two terms of Equation (3) around $\mathbf{r}_{\mathrm{m}}$, we find that the zero orders cancel out and we obtain

$$
\ddot{\tilde{\mathbf{r}}}=-\frac{G M}{r_{\mathrm{m}}^{3}} \tilde{\mathbf{r}}+3 \frac{G M}{r_{\mathrm{m}}^{5}}\left(\tilde{\mathbf{r}} \mathbf{r}_{\mathrm{m}}\right) \mathbf{r}_{\mathrm{m}}-\frac{G m}{\tilde{r}^{3}} \tilde{\mathbf{r}} .
$$

Rescaling the distance between the bodies by $(m / M)^{1 / 3} r_{p}$ and the time by $\sqrt{r_{p}^{3} / G M}$, we can re-write Equation (6) in terms of the dimensionless variables ${ }^{5} \mathbf{r}$ and $t$ :

$$
\ddot{\mathbf{r}}=\left(\frac{r_{p}}{r_{\mathrm{m}}}\right)^{3}\left[-\mathbf{r}+3\left(\mathbf{r} \hat{\mathbf{r}}_{\mathrm{m}}\right) \hat{\mathbf{r}}_{\mathrm{m}}\right]-\frac{\mathbf{r}}{r^{3}} .
$$

Since $\hat{\mathbf{r}}_{\mathrm{m}}=(\cos f, \sin f, 0), r_{\mathrm{m}} / r_{\mathrm{p}}=2 /(1+\cos f)$, and we set $\mathbf{r}=(x, y, z)$, explicit equations in terms of dimensionless Cartesian coordinates read

$$
\begin{aligned}
& \ddot{x}= \frac{(1+\cos f)^{3}}{8}[-x+3(x \cos f+y \sin f) \cos f] \\
&-\frac{x}{\left(x^{2}+y^{2}+z^{2}\right)^{3 / 2}}, \\
& \ddot{y}= \frac{(1+\cos f)^{3}}{8}[-y+3(x \cos f+y \sin f) \sin f] \\
&-\frac{y}{\left(x^{2}+y^{2}+z^{2}\right)^{3 / 2}}, \\
& \ddot{z}=-\frac{(1+\cos f)^{3}}{8} z-\frac{z}{\left(x^{2}+y^{2}+z^{2}\right)^{3 / 2}}, \\
& t=\frac{\sqrt{2}}{3} \tan (f / 2)\left(3+\tan ^{2}(f / 2)\right) .
\end{aligned}
$$

\footnotetext{
5 Later, for the radial problem (Section 3), we will rescale our variables differently, keeping the same symbols. Therefore, throughout this paper, $\mathbf{r}$ and $t$ should be understood as dimensionless, with a normalization that should be clear from the context.
} 
Equations (8)-(10) are the equivalent of the Hill equations (Hill 1886), for the parabolic rather than circular problem. Since Equation (11) is implicit, for numerical applications it may be preferable to use its differential form

$$
\dot{f}=\sqrt{2}(1+\cos f)^{2} / 4 .
$$

\subsection{Free Solutions}

Just like the Hill equations, Equations (8)-(10) have free solutions, those that ignore the interaction term $\mathbf{r} / r^{3}$. These can be found mathematically, but physical intuition facilitates a swift solution. Since we have a set of three linear differential equations of the second order, all solutions are a linear combination of six independent solutions. Each could be physically obtained by taking the difference between an orbit infinitesimally close to a parabolic orbit and the parabolic orbit itself. We list the solutions below, stating which orbital element has been varied.

1. Variation in the argument of periapsis:

$$
x=-\frac{\sin f}{1+\cos f}, \quad y=\frac{\cos f}{1+\cos f} .
$$

2. Variation in the time of periapsis:

$$
x=-\sin f, \quad y=1+\cos f .
$$

3. Variation in the periapsis distance:

$$
x=2-\cos f, \quad y=-\cos f \tan (f / 2) .
$$

4. Variation in the eccentricity at fixed periapsis:

$$
\begin{gathered}
x=(8+12 \cos f) \tan ^{4}(f / 2), \\
y=\frac{35 \sin f-2 \sin (2 f)+3 \sin (3 f)}{(1+\cos f)^{2}} .
\end{gathered}
$$

5. Rotation around the apsidal line:

$$
x=y=0, \quad z=\frac{2 \sin f}{1+\cos f} .
$$

6. Rotation around the latus rectum:

$$
x=y=0, \quad z=\frac{2 \cos f}{1+\cos f} .
$$

In the above expressions, $f$ is a function of $t$ as given by Equation (11). The first four solutions are planar, i.e., $z=0$, while the last two solutions are one dimensional, $x=y=0$. It is easy to check the validity of these expressions by substituting them into Equations (8)-(10) and using Equation (12).

\subsection{The Energy}

We are ultimately interested in the fate of a star in a binary, following its encounter with the BH. If the binary - approaching the hole on a parabolic orbit-is torn apart, a star can either become bound to the $\mathrm{BH}$ or be ejected from the system. To distinguish between these two possibilities, we calculate its energy as a function of time, including the negative gravitational energy due to the BH. Initially, at large distances, the specific energy of one member is simply $\sim-v_{0}^{2}$. After the binary disruption, the analytical arguments in our introduction suggest that its energy is larger by a factor of $(M / m)^{1 / 3} \gg 1$. We thus neglect the term due to the self-gravity of the binary. In addition, for $(M / m) \gg 1$, the change in energy of the massive $\mathrm{BH}$ can also be neglected and the total energy of $m_{1}$ reads

$$
E_{1}=-\frac{G M m_{1}}{r_{1}}+m_{1}\left|\dot{\mathbf{r}}_{1}\right|^{2} / 2 .
$$

To zeroth order, we can replace $\mathbf{r}_{1}$ by $\mathbf{r}_{\mathrm{m}}$, but since $r_{\mathrm{m}}$ is a parabolic orbit that zeroth-order energy vanishes. The first-order terms are

$$
E_{1}=\frac{G M m_{1}}{r_{\mathrm{m}}^{2}}\left(r_{1}-r_{\mathrm{m}}\right)+m_{1} \dot{\mathbf{r}}_{\mathrm{m}}\left(\dot{\mathbf{r}}_{1}-\dot{\mathbf{r}}_{\mathrm{m}}\right),
$$

or, using our rescaled variables,

$$
E_{1}=-\frac{G M m_{1} m_{2}}{m r_{p}}\left(\frac{m}{M}\right)^{1 / 3}\left[\frac{r_{p}^{2}}{r_{\mathrm{m}}^{2}} \hat{\mathbf{r}}_{\mathrm{m}}+\frac{\dot{\mathbf{r}}_{\mathrm{m}}}{r_{p}} \mathbf{\mathbf { r }}\right] .
$$

Since in our limit the total energy of the system is zero, the energy of the other body, $m_{2}$, is simply $E_{2}=-E_{1}$. In the following, it is useful to define the penetration factor $D=r_{p} / r_{t}$. In terms of our dimensionless Cartesian coordinates Equation (21) is given by

$$
\begin{gathered}
E_{1}=-E_{2}=-\frac{G m_{1} m_{2}}{a D}\left(\frac{M}{m}\right)^{1 / 3} \\
\times\left[\frac{(1+\cos f)^{2}}{4}(x \cos f+y \sin f)+\frac{-\sin f \dot{x}+(1+\cos f) \dot{y}}{\sqrt{2}}\right] .
\end{gathered}
$$

If the binary dissolves, this energy tends to a constant, since the body is eventually moving only under the conservative force of the BH. Mathematically, this means that the first term on the right-hand side of Equation (7) now dominates, and the problem is linear.

The negative of a solution is thus a solution. But the energy is also linear in the coordinates. Therefore, a body starting with a phase difference $\pi$ will have the same final energy in absolute value but opposite in sign. This is independent of the mass of the star. The important consequence is that, of the disrupted cases, half would have the heavier object bound and the lighter escaping, while half would have the opposite. These findings are at odds with those of Bromley et al (2006) who find that, for large $m_{1} / m_{2}$ ratios, the lighter object usually becomes bound.

We also note that, to this lowest order, the $z$-component of the binary motion has no effect on the energy; thus it does not determine whether a body would be ejected.

Finally, we explicitly write the energy for the case that we can neglect the interaction term between the two stars. The solution in this "free" regime ${ }^{6}$ is a linear combination of Equations (13)(18). For each free solution the energy is a constant of motion. Therefore, the total energy is a linear combination of these constants. However, any solution constructed from two bodies in infinitesimally close parabolic orbits has zero energy. Only Equation (16), that describes the relative orbits of two bodies where one has $e \neq 1$, gives a finite constant energy when substituted into Equation (22):

$$
E_{1}=-E_{2}=-\frac{G m_{1} m_{2}}{a D}\left(\frac{M}{m}\right)^{1 / 3} 20 B,
$$

\footnotetext{
6 Even in the non-free regime, where the gravitational forces between the two stars are important, one can expand the solutions in terms of the free solutions, except that the coefficients will be time dependent. Our expression of the energy will still be given by $B$, except that $B$ now is time dependent.
} 
where $B$ is the coefficient of Equation (16) in the linear expansion.

\section{DEEP PENETRATORS AND THE RADIAL RESTRICTED THREE-BODY PROBLEM}

The previous section allowed for arbitrary penetration factors $D$. The presence of the factor $1 / D$ in Equation (23) seems to suggest that, for a given initial star separation $a$, arbitrarily large energies can be attained for binary trajectories penetrating far into the tidal disruption region $(D \ll 1)$. However, as we show here, this is not the case. We proceed to investigate this case of deep penetrators by considering a different, even simpler, limit of the equations. The periapsis distance of the center of mass, $r_{p}$, becomes now irrelevant and instead the center of mass can be taken to move on a radial orbit given by

$$
r_{\mathrm{m}}=\left(\frac{9 G M \tilde{t}^{2}}{2}\right)^{1 / 3}
$$

We can now scale the distance to the initial semimajor axis of the binary, $a$, and the time to the inverse angular frequency of the binary with that semimajor axis, $\sqrt{G m / a^{3}}$. Taking the direction of the binary center of mass with respect to the $\mathrm{BH}$ to be $\hat{\mathbf{r}}_{\mathrm{m}}$, Equation (6) simplifies to

$$
\ddot{\mathbf{r}}=\left(\frac{2}{9 t^{2}}\right)\left[-\mathbf{r}+3\left(\mathbf{r} \hat{\mathbf{r}}_{\mathrm{m}}\right) \hat{\mathbf{r}}_{\mathrm{m}}\right]-\frac{\mathbf{r}}{r^{3}} .
$$

In Cartesian coordinates, where $\hat{\mathbf{x}}$ is the direction of the binary's center of mass, we have

$$
\begin{aligned}
\ddot{x} & =\frac{4}{9 t^{2}} x-\frac{x}{\left(x^{2}+y^{2}+z^{2}\right)^{3 / 2}}, \\
\ddot{y} & =-\frac{2}{9 t^{2}} y-\frac{y}{\left(x^{2}+y^{2}+z^{2}\right)^{3 / 2}}, \\
\ddot{z} & =-\frac{2}{9 t^{2}} z-\frac{z}{\left(x^{2}+y^{2}+z^{2}\right)^{3 / 2}} .
\end{aligned}
$$

The energy as given by Equation (20) is

$$
E_{1}=-E_{2}=-\frac{G m_{1} m_{2}}{a}\left(\frac{M}{m}\right)^{1 / 3}\left(\frac{2}{9}\right)^{2 / 3}|t|^{-4 / 3}(x+3 t \dot{x}) \text {. }
$$

\subsection{Special Solutions to the Radial Restricted Problem}

\subsubsection{Homogeneous Collapse}

It is simple to verify that the following

$$
x(t)= \pm\left(\frac{3}{2}\right)^{1 / 3}|t|^{2 / 3}, \quad y(t)=z(t)=0
$$

is a solution to our equations. This solution is the analog of a homogenous collapse. In these solutions, the small masses $m_{1}$ and $m_{2}$ accelerate toward each other, by a combination of tidal forces and mutual acceleration, at the same rate that their center of mass is accelerating toward the central mass. These solutions, just like the stationary solution at the $L_{1}$ and $L_{2}$ points in the circular restricted problem, and just like the homogeneous collapse of dust, are unstable for deviations in the $x$-direction.

\subsubsection{Free Solutions}

In the limit of large separation between the small masses or close to $t=0$, where the distance to the $\mathrm{BH}$ is small, tides dominate over the mutual gravity, and the problem become separable in $x, y$, and $z$. It admits the following solutions:

$$
\begin{aligned}
& x(t)=A_{x}|t|^{-1 / 3}+B_{x}|t|^{4 / 3}, \\
& y(t)=A_{y}|t|^{1 / 3}+B_{y}|t|^{2 / 3}, \\
& z(t)=A_{z}|t|^{1 / 3}+B_{z}|t|^{2 / 3} .
\end{aligned}
$$

These are the equivalent of the free solutions given for the parabolic restricted problem in Equations (13)-(18). Just like them, they can be given physical interpretations. The $A_{x}$ solution describes two particles that have the same trajectory, but are slightly separated in time. The $B_{y}$ and $B_{z}$ solutions describe particles going on slightly different radial paths, each of zero energy. The $B_{x}$ solution describes the relative orbits of two particles going on the same radial path, but with slightly different energies. The $A_{y}$ and $A_{z}$ solutions describe the relative orbits of particles with slightly different angular momentum.

Note that both exponents of the $y$ and $z$ terms are positive, while one exponent of the $x$ term is negative. This means that at times close to zero, the particles are very close in $y$ and $z$ but are separated in $x: y(t \rightarrow-0)=z(t \rightarrow-0)=0$ while $x(t \rightarrow-0)= \pm \infty$.

As in the parabolic case, one of the coefficients of the solution is related to energy. Substituting the equation for the $x$-component and its derivative into Equation (29), we get

$$
E_{1}=\frac{G m_{1} m_{2}}{a}\left(\frac{M}{m}\right)^{1 / 3} 5(2 / 9)^{2 / 3} B_{x}
$$

This can be regarded as the equivalent of Equation (23) in the limit of $D \rightarrow 0$. It is evident that the energy in this limit is finite and the divergence suggested by Equation (23) is not real. Moreover, as we will show later, the highest ejection velocities are not obtained in this limit.

$$
\text { 3.2. The } t=0 \text { Singularity }
$$

From the discussion above, we learn that, beside a set of zero measures of initial conditions, the binary components acquire an increasingly large separation as they approach the large mass $M$. It is therefore tempting to conclude that, for deep penetrators, binaries always dissolve. In fact, the two components of the binary approach each other again at later (positive) times. Thus, to know the final outcome of the scattering, we should overcome the singularity and follow the orbits of the light bodies beyond the time of the encounter with the $\mathrm{BH}$.

The difficulty arises from the assumption of a purely radial orbit for the binary center of mass. A deep $(D \ll 1)$ parabolic orbit would parallel closely the radial one and get around the $\mathrm{BH}$ smoothly. Once across the singular region, the radial equations are valid and the integration of the orbit can be resumed. We further notice that when the binary is well within the tidal radius, $|t| \ll 1$, the mutual gravity of its members can be neglected and their separation follows a free solution. Therefore, the resolution of our problem ultimately lies in finding which free parabolic orbit reduces - in the deep penetration approximation - to the free radial orbit that an approaching binary is following while still farther away from its periapsis, $|t| \gg D^{3 / 2}$. 
This translates into expanding Equations (13)-(18) around $f=-\pi$ and comparing them with Equations (31). Interestingly, we find that there is a one to one correspondence: solutions with coefficients $A_{x}, A_{y}$, and $A_{z}$ correspond to Equations (14), (15), and (17), respectively. Likewise, the solutions with $B_{x}$, $B_{y}$, and $B_{z}$ correspond to Equations (16), (13), and (18), respectively. This is as expected, given their physical interpretation. As an example, we expand Equation (11) and the $x$-component of Equation (16) multiplied by some coefficient $B$ around $f=-\pi$. We obtain

$$
x=-6^{4 / 3}|t|^{4 / 3} B .
$$

This is the behavior of the radial solution with coefficient $B_{x}$. In fact, comparing the two solutions for $x$ and accounting for the different spatial and temporal dimensions, we get

$$
B_{x}=-6^{4 / 3} D^{-1} B .
$$

With this relation, the two Equations (23) and (32) are equivalent, as we expect.

From this comparison, we also learn the behavior of the binary separation around $t=0$. There, the $A_{x}|t|^{-1 / 3}$ term dominates, corresponding to the free parabolic solution derived by slightly varying the time at periapsis (Equation (14)). The latter, however, does not diverge at $t=0$. Instead, it describes a circle centered around $x=0$ with $y=R$, where $R$ is the radius of the circle.

A key observation for our problem is that all parabolic orbits which reduce to the $A_{s}\left(B_{s}\right)$ solutions are asymmetric (symmetric) function of time where the subscript $s$ stands for $x, y$, or $z$. We conclude that we can ferry a free solution across $t=0$, from negative to positive times, by simply changing the sign of its $A_{s}$ coefficients.

A concern might arise from the fact that our assumption of small binary separation relative to the BH may break down at times where the binary is close to its periapsis. Indeed, at small enough times, the binary separation is about $a|t|^{-1 / 3}$ while the distance to the BH decreases as $r_{\mathrm{m}} \sim a(M / m)^{1 / 3}|t|^{2 / 3}$. They match at $|t|=(m / M)^{1 / 3}$, from which follows that, if the binary gets within a distance of $r_{\mathrm{m}} / a<(m / M)^{1 / 9}$ from the $\mathrm{BH}$, our approximation is no longer valid. This occurs for a penetration factor for the orbit smaller than $D<(m / M)^{2 / 9}$. When this happens, the parabolic formalism also incorrectly traces the trajectory for $|t|<(m / M)^{1 / 3}$. Specifically, the true orbit deviates from the circle given by Equation (14). Yet, the rest of our conclusions, including the energy of the particles, will not be affected. Using the same work argument in our introduction, one derives that the energy gained during the periapsis passage is smaller by a factor of $D^{2}$ than that gained around the tidal radius. One factor of $D$ come from the smaller distance around the BH, and the other from the larger distance between the stars resulting in a smaller force between them. We conclude that-for any impact parameter-both energy and orbit obtained with the method described above are fairly accurate for $|t|>(m / M)^{1 / 3}$.

\section{NUMERICAL VERIFICATIONS}

We test our approximated equations against three-body simulations of a binary evolving around a much more massive BH.

Both the three-body code and the code that numerically integrates our equations are provided with a fourth-order RungeKutta integration scheme. In the three-body code, the binary center of mass is initially either on a parabolic (Section 4.1) or

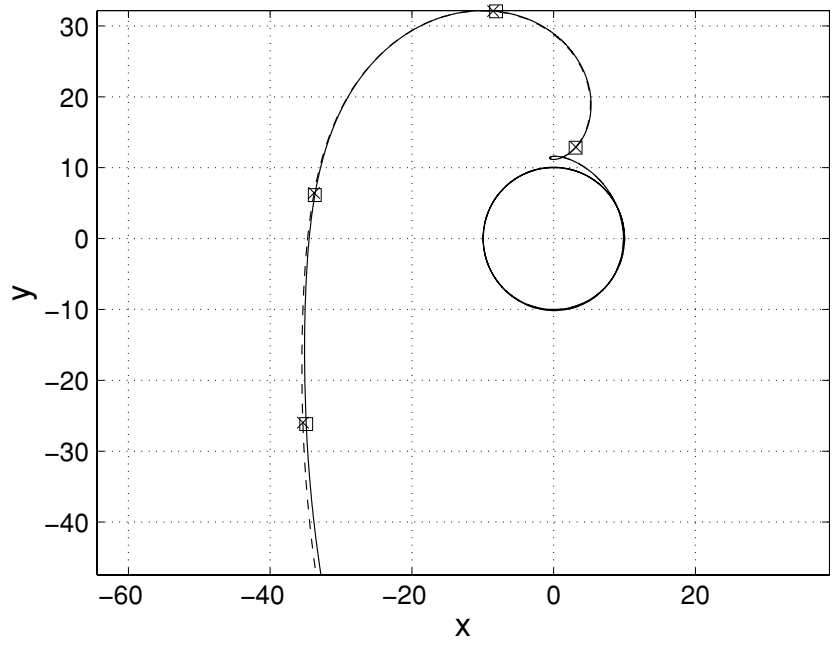

Figure 1. Orbit of the secondary star in the primary comoving frame. The binary center of mass is initially on a parabolic trajectory. We assume a prograde binary orbit, $M / m=10^{6}, m_{1} / m_{2}=3, D=0.1, \phi=4 \pi / 5$, and $r_{0}=10 r_{t}$. The three-body solution (solid line) is compared with our approximated solution (dashed line). Squared (three body) and cross (our solution) marks show the position of the secondary at $t=-3,0,3$, and 15 . Lengths and time are in units of $(m / M)^{1 / 3} r_{p}$ and $\left(r_{p}^{3} / G M\right)^{1 / 2}$, respectively.

on a radial (Section 4.2) orbit. The binary consists of a primary star with mass $m_{1}$ and of a secondary with $m_{2}=m_{1} / 3$. In the examples shown in this section, the BH-binary mass ratio is set to be $M / m=10^{6}$. The binary's orbit is assumed to be initially circular in the comoving frame of the binary center of mass. The initial configuration of the system is characterized by three parameters. First, the initial distance of the binary center of mass to the $\mathrm{BH}, r_{0}$. However, as long as a simulation starts at a large enough radius, $r_{0} \gg r_{\mathrm{t}}$, the orbits are largely independent of it. In our runs, we assume $r_{0}=10 r_{t}$, which is sufficient for convergency. Second, the initial (at $t_{0}=t\left(r_{0}\right)<0$ ) binary phase, $\phi_{0}$. We parameterize it using the effective phase $\phi$ at $t=0$ (i.e., at the periapsis passage for a parabolic orbit or at $r=0$ for a radial one),

$$
\phi_{0}=S \omega t_{0}+\phi,
$$

where $\omega$ is the (constant) angular velocity of the binary at $r \gg r_{t}$. Naturally, the actual phase at $t=0$ is in general different from $\phi$, due to the BH tidal forces. All angles are measured from the $x$-axis. Finally, we should specify the direction of rotation of the binary, $S$, as viewed in the non-rotating frame: i.e., the relative orientation of the angular momentum of the binary around the $\mathrm{BH}$ and of a star around the binary center of mass. For a planar orbit there are two possibilities: the angular momenta are aligned, $S=1$, in which case we call the orbit prograde, or they are anti-aligned, $S=-1$, which we call a retrograde orbit. In addition, for the parabolic case, the system has a fourth parameter: the penetration factor $D$.

\subsection{Parabolic Orbits}

In this section, we compare the evolution of the binary stars obtained with the three-body code and our parabolic formalism (Equations (8)-(11)). We assume a prograde orbit for the binary, $D=0.1$ and $\phi=4 \pi / 5$. The result is shown in Figure 1, where we plot the orbit of the secondary star in the comoving frame of the primary. After disruption around periapsis, the secondary star is captured by the $\mathrm{BH}$ on an elliptical orbit, while the primary is ejected from the system. Clearly, the three-body curve is accurately reproduced by our set of approximated equations. 


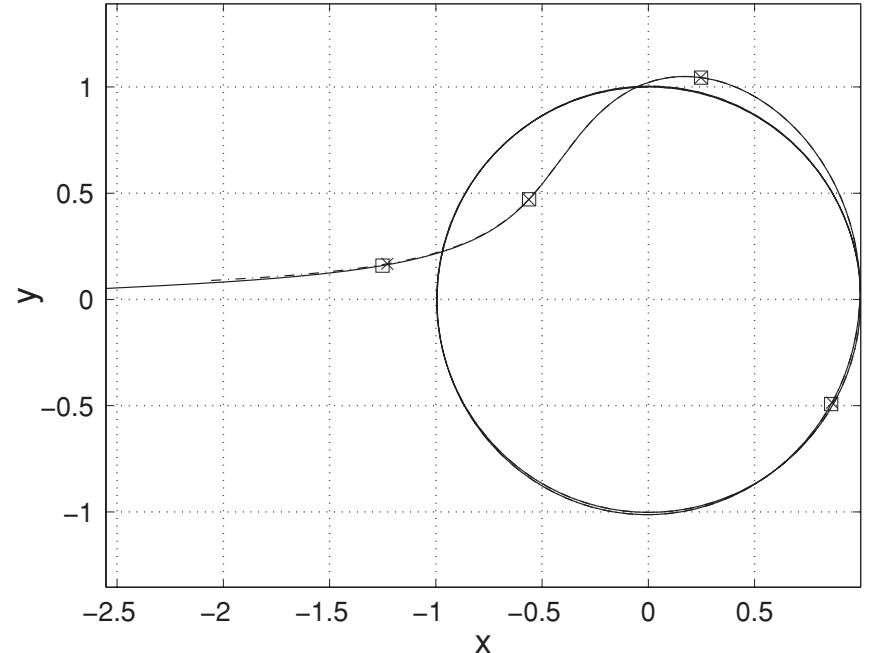

Figure 2. As Figure 1, but for an initial radial orbit for the binary center of mass. All relevant parameters are the same. Here, the position of the secondary is marked at $t=-10^{-2},-10^{-1},-1$, and -3 . Length and time are in units of $a$ and $\sqrt{a^{3} / G m}$, respectively.

The energy, for example, differs at a $0.1 \%$ level after the binary disruption.

In addition, we compared the performance of the two numerical methods for $M / m \gg 1$. The calculation of our approximated solution is faster for two reasons. First, for the fourth-order scheme, numerical convergence is achieved with a time-step about $\sim(M / m)^{1 / 12}$ times smaller. Second, we integrate seven equations instead of eighteen, since we only follow the binary star's relative distance. This moderate speed-up, combined with the fact that most dependencies are analytic, allows us to explore more easily a wider portion of the parameter space.

\subsection{Radial Orbits}

We now consider a binary moving on a radial orbit of zero energy and test the accuracy of our radial approximation (Equations (26)-(28)). The trajectory of the separation between the stars is shown in Figure 2 for a binary with a phase $\phi=4 \pi / 5$. Here, we plot the orbit up to $t=-2 \times 10^{-3}$. The assumption of a relatively small star separation is expected to become invalid around $|t| \sim(m / M)^{1 / 3}=10^{-2}$. Again, there is good agreement between our solution and the three-body calculation. Comparing the two terms on the right-hand side of Equation (26), one expects that the tidal force dominates for $|t| \ll 1$. Indeed, we find numerically that the deviation from the initially circular orbit becomes significant around $|t| \sim 1$. For $|t| \ll 1$, the orbit approaches the free solutions $x \propto|t|^{-1 / 3}$ and $y \propto|t|^{1 / 3}$. Finally, we also reproduce at a percentage level the energy evolution as a function of time. For the specific example in Figure 2, we get an energy that differs only by half a percent at $t \sim 0$ from the three-body result.

\subsection{Deep Penetrators}

In the previous sections, we have established that both our sets of equations reproduce the full three-body calculation well. We now investigate their relationship. In particular, we show how a parabolic solution reduces to a radial one in the limit in which a binary plunges deeply into the tidal sphere of influence of the BH. With this aim, we employ our parabolic formalism to numerically calculate binary orbits with increasingly smaller

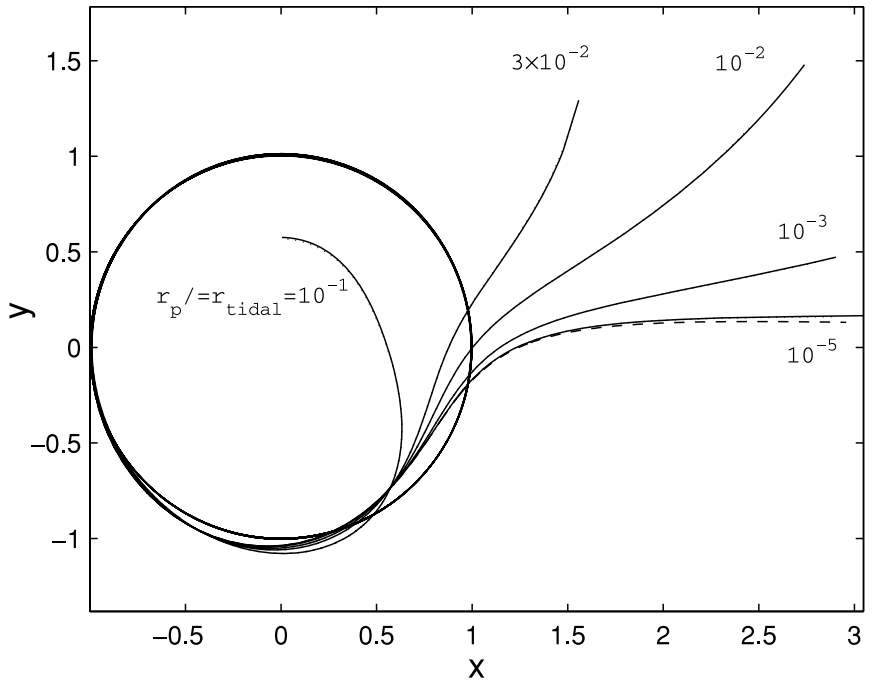

Figure 3. As Figure 1, but for different penetration factors as labeled. The binary's orbit is prograde with parameters $M / m=10^{6}, \phi=0$, and $r_{0}=$ $10 r_{t}$. Lengths are in units of the initial binary separation. The parabolic approximation (solid curves) and the three-body results (dotted curves) are practically indistinguishable. In addition, we plot the radial orbit (dashed curve) for the same parameters.

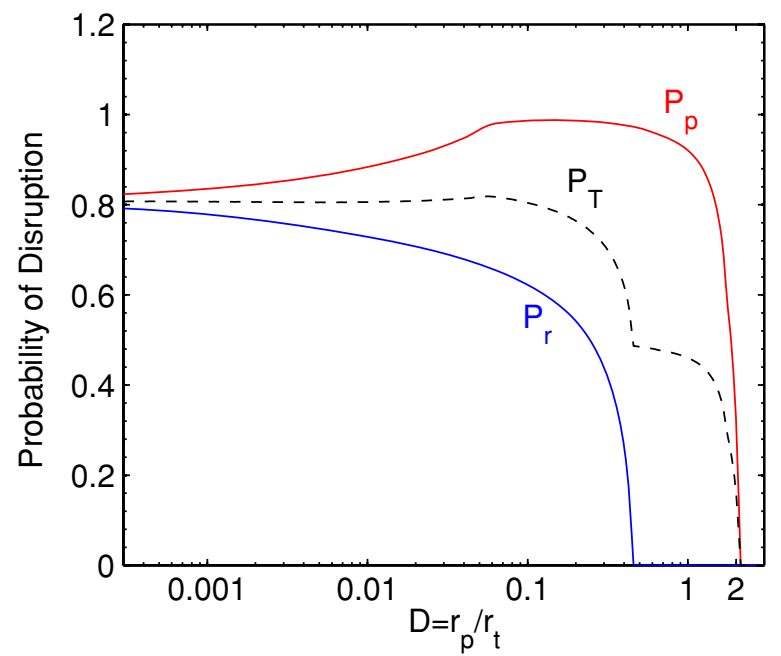

Figure 4. Probability of disruption as a function of the penetration factor, $D$, for prograde orbit $\left(P_{\mathrm{p}}\right.$, the red solid line $)$, retrograde orbit $\left(P_{\mathrm{r}}\right.$, the blue solid line), and total $P_{\mathrm{T}}=\left(P_{\mathrm{p}}+P_{\mathrm{r}}\right) / 2$. The binary phase $[0, \pi]$ is sampled with 3000 equally spaced grid points for each penetration factor.

(A color version of this figure is available in the online journal.)

penetration factors (Figure 3, solid lines). In this example, the orbit degenerates to a radial one (dashed line) for $D \sim 10^{-5}$.

\section{RESULTS}

We are now in the position to statistically describe the properties of a binary after its encounter with a BH. We focus here on the planar case. For given masses of the three bodies and semimajor axis of the binary stars, the fate and the final energy of the binary members depend only on the penetration factor $D=r_{p} / r_{t}$ and on the effective binary phase $\phi$.

\subsection{The Fate of the Binary}

Contrary to naive expectations, we find that for $D \ll 1$ a nonnegligible fraction of the binaries are not disrupted (Figure 4). For $D<10^{-1}$, the fraction of disrupted cases saturates at a level of $\sim 80 \%$. The best chance of disruption is for a binary 

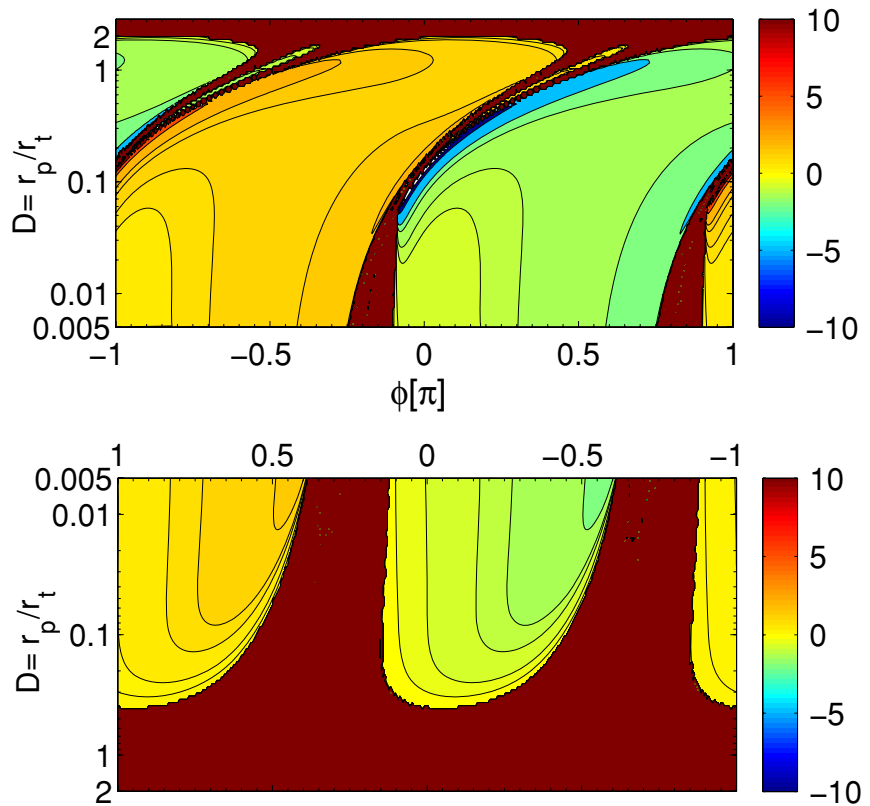

Figure 5. Final energy contour plots, in the phase-penetration factor plane, for a given initial separation $a$. Upper panel: prograde orbits. Lower panel: retrograde orbits. Energy is in units of $\left(G m_{1} m_{2} / a\right)(M / m)^{1 / 3}$ and the phase in units of $\pi$. In the lower panel axes are reversed. The energies have been computed using the restricted parabolic formalism. The figure is constructed with a grid of $400 \phi$ values (equally spaced linearly) and $100 \mathrm{D}$ values (equally spaced logarithmically). We only plot the final energy of a member of a disrupted binary, which can be either captured by the $\mathrm{BH}(E<0)$ or ejected $(E>0)$. The flat amaranth (dark red) region shows the region in the parameter space where the binary survives disruption.

in prograde orbit with $D=0.15$. A comparison between the curves for prograde (labeled $\left.P_{\mathrm{p}}\right)$ and retrograde $\left(P_{\mathrm{r}}\right)$ orbits underlines the well known fact that retrograde binaries tend to be more stable against tidal disruption. Nevertheless, the two curves converge for $D \ll 1$. This is because in this limit the binary center of mass approaches the $\mathrm{BH}$ in an almost radial fashion, so that the angular momentum of the binary around the $\mathrm{BH}$ is very close to zero. Indeed, in the radial formalism, there is no distinction between prograde and retrograde orbits. Consistently, when we calculate the disruption probability for a binary on a radial orbit, we find a survival fraction of $19 \%$. Interestingly, we observe that binaries that avoid disruption tend to tighten, with their final semimajor axis $a_{\mathrm{f}}<a$. For instance, for radial orbits, the encounter with the $\mathrm{BH}$ produces harder binaries in about $90 \%$ of the cases.

\subsection{Final Energy of Ejected Stars}

In Figure 5, we show the contour plots for the final energy, at $t \gg 1$, of a member of a binary in parabolic orbit that faces either capture by the $\mathrm{BH}(E=$ constant $<0)$ or ejection $(E=$ constant $>0)$ from the three-body system. The flat amaranth region is where binaries are not disrupted. For a binary approaching the $\mathrm{BH}$ on a prograde orbit (upper panel), a rather shallow penetration factor of $D=2.1$ is already sufficient to be torn apart by tidal forces, while no disruption occurs for a retrograde orbit (lower panel) with $D \geqslant 0.44$. We chose to plot the lower panel with reversed axes to emphasize how they tend towards the same energy distribution for $D \rightarrow 0$. The limiting distribution depends only on $\phi$ and it is given, of course, by the energy plot obtained for radial orbits (Figure 6).

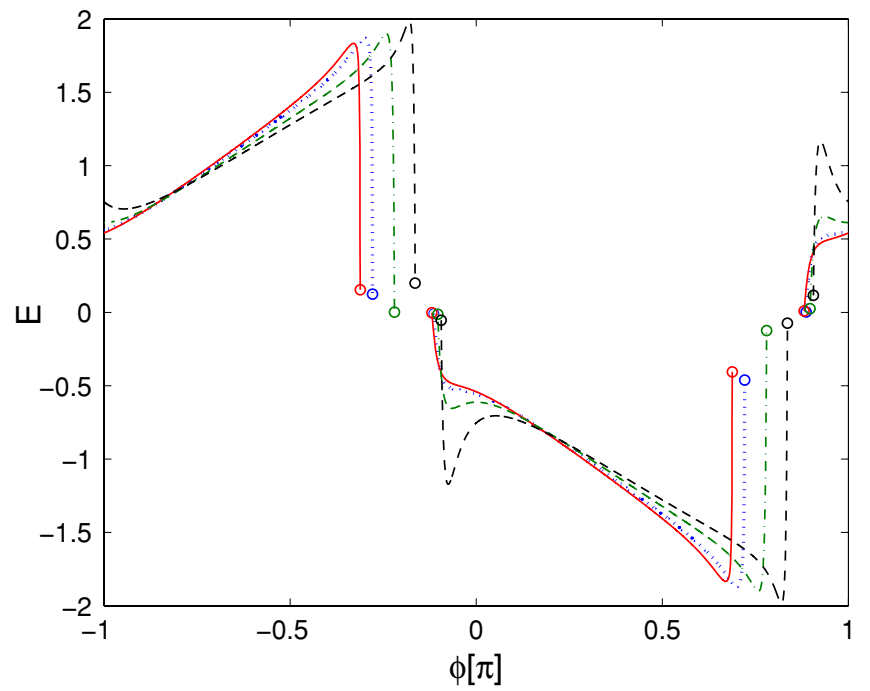

Figure 6. Final energy as a function of $\phi$ for a member of a deep penetrating disrupted binary with a given initial separation $a$. Energy is in units of $\left(G m_{1} m_{2} / a\right)(M / m)^{1 / 3}$ and the phase in units of $\pi$. The red solid line is for a binary in radial orbit. Results for the parabolic formalism are shown for prograde orbits with $D=10^{-3}$ (blue dotted line), $10^{-2}$ (green dash-dotted line), and $3 \times 10^{-2}$ (black dashed line). The boundaries of the intervals in $\phi$, where disruption of the binary occurs, are marked with the circles. The phase range is sampled with 6000 equally spaced grid points.

Note again that in the radial limit there is a finite region $(-0.31 \pi<\phi<-0.12 \pi$ and $0.69 \pi<\phi<0.88 \pi)$ where binaries survive.

Let us now consider the pattern of the energy contours (Figures 5 and 6). For the same penetration factor, it shows how a change of $\pi$ in the effective phase translates into an energy which is equal in absolute value but opposite in sign. Therefore, as noted before, a uniform distribution in $\phi$ implies that, when the binary is disrupted, each body has a $50 \%$ chance of being ejected. Another noticeable feature is the presence and position of steep narrow peaks next to large plateaux. For prograde orbits, peaks of $|E| \simeq 27$ are attained not in the radial limit but for a finite penetration factor $D \simeq 10^{-1}$ (see also the solid red curve in the lower panel of Figure 7). On the other hand, for retrograde orbits, peaks - or rather "hills"-of a more modest maximum energy of $|E| \simeq 1.8$ emerge gradually as $D$ gets smaller (see the red dashed curve, same panel). As well as the maximal ejection energy, we also plot in Figure 7 the ejection energy averaged over the binary phase as a function of $D$ (upper panel). In both plots, a rather large peak is present only for prograde orbits (red solid curves) and it is situated in the range $10^{-1}<D<1$.

A brief summary of the quantitative results of this section follows.

1. The largest $D$ for which there is disruption is $D=2.1$ for prograde orbits and $D=0.44$ for retrograde orbits.

2. The maximum energy is 27.3 for prograde orbits and 1.8 for retrograde orbits.

3. The average energy is 1.46 for prograde orbits, 0.50 for retrograde orbits, and 1.36 for prograde and retrograde orbits together. In averaging, we have assumed that $D$, or equivalently $r_{p}$, is uniformly distributed.

4. The highest chance for disruption is for prograde orbits with $D=0.15$. 

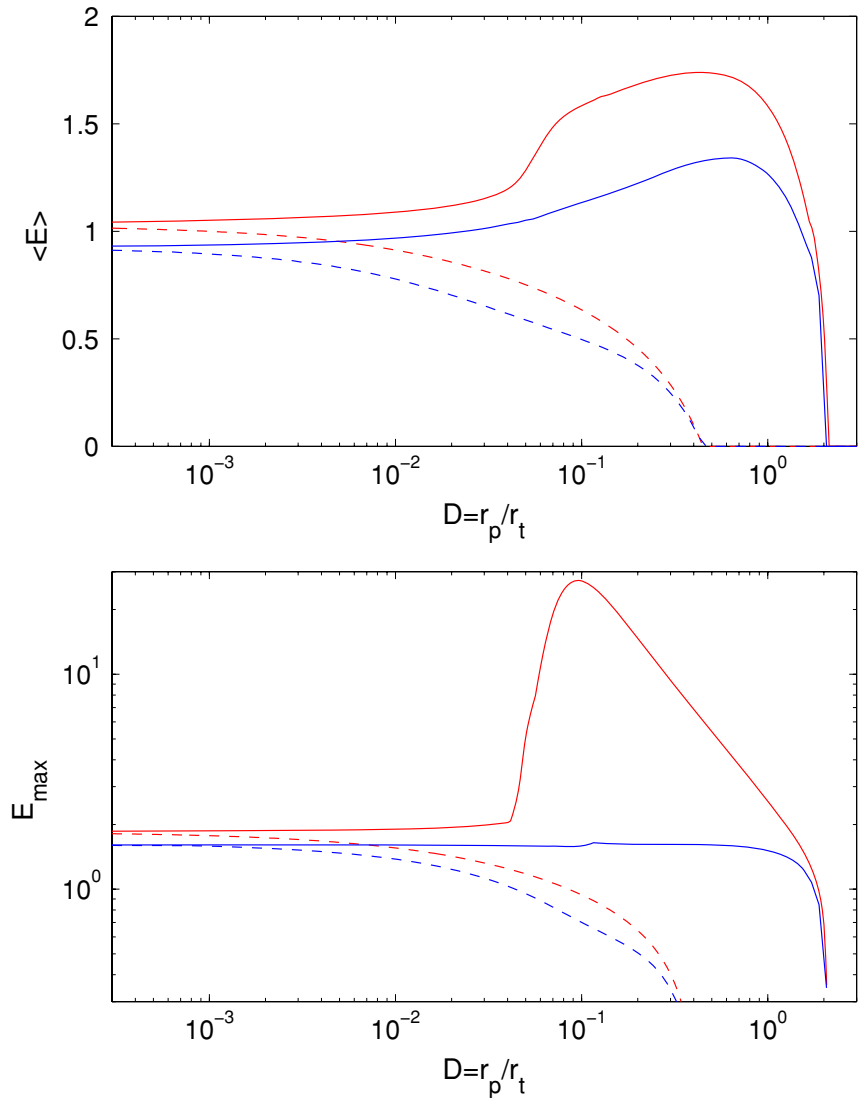

Figure 7. Top panel: ejection energy averaged over binary phase as a function of the penetration factor. The solid (dashed) line is for prograde (retrograde) orbits. The energies for a binary with a given $a$ are plotted as red lines, in units of $\left(G m_{1} m_{2} / a\right)(M / m)^{1 / 3}$. The energies for a binary with a given $R_{\min }$ are plotted as blue lines, in units of $\left(G m_{1} m_{2} / R_{\min }\right)(M / m)^{1 / 3}$. Bottom panel: as upper panel, but for the maximum ejection energy for a given $D$ as a function of $D$. The average and maximum values are evaluated for the absolute value of the energy $|E|$.

\section{FINITE STELLAR SIZES}

Up until now, we have ignored the finite size of the stars. However, the highest energies or ejection velocities are obtained when stars, under the action of the $\mathrm{BH}$ tides, get closer to each other before the binary dissolves. Specifically, note the sharp peaks in Figure 7 (lower panel) with exceptional high final energy. Inspection of these orbits reveals that the minimal separation that these binaries attain over their evolution is more than ten times smaller than their initial separation. Those orbits, however, are not always physical: if two stars start as almost contact binaries and contract further, they may collide and merge or tidally disrupt each other. We therefore consider in the following a given minimum separation $R_{\min }$ - about the sum of the radii of the two stars-below which a binary cannot shrink.

The parabolic and radial formalisms discussed above provide the relative trajectory of the two stars, but the dimensions are arbitrary and can be rescaled. For each of these trajectories, we can find the minimum dimensionless distance $\left.\sqrt{x^{2}+y^{2}+z^{2}}\right|_{\min }$ over the whole orbit, and scale it to be equal to $R_{\min }$. This amounts to deriving the initial binary separation results in orbits whose minimal separation is $R_{\min }$ : $a=R_{\min } /\left.D \sqrt{x^{2}+y^{2}+z^{2}}\right|_{\min }$ in the parabolic formalism, and $a=R_{\min } /\left.\sqrt{x^{2}+y^{2}+z^{2}}\right|_{\min }$ in the radial formalism.
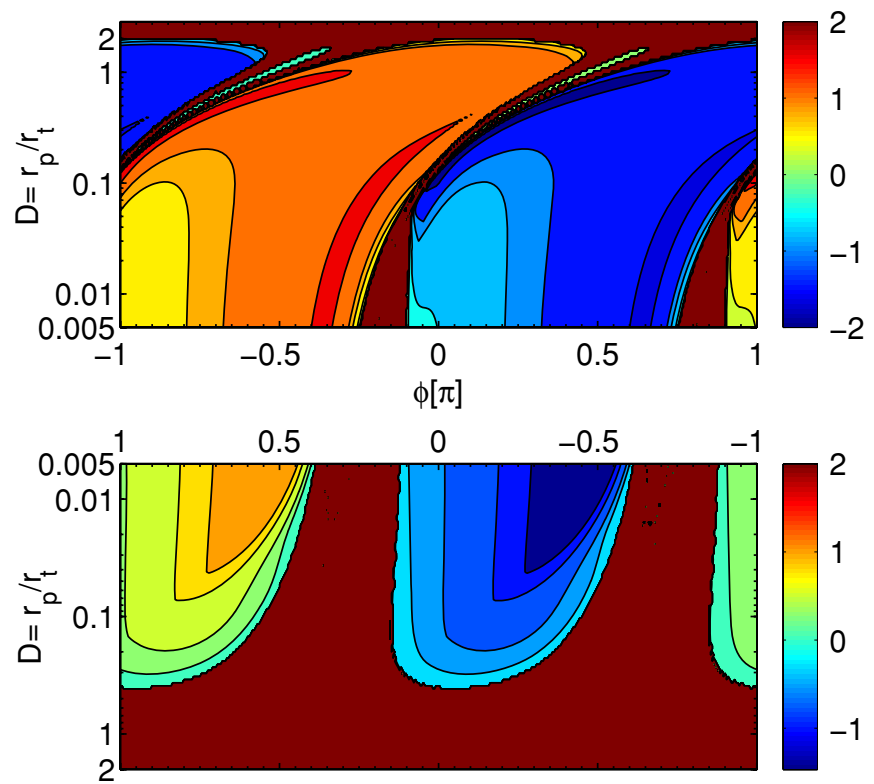

Figure 8. As Figure 5 but for a given minimal distance $R_{\min }$. Here, energy is in units of $\left(G m_{1} m_{2} / R_{\min }\right)(M / m)^{1 / 3}$ and the phase in units of $\pi$. Note the energy scale, from -2 to 2 , a factor of 5 smaller than the energy scale needed for prograde orbits with a fixed $a$ (Figure 5, upper panel). by

Assuming this $a$ as our initial condition, the energy is given

$$
\begin{gathered}
E_{1}=-E_{2}=-\left.\frac{G m_{1} m_{2}}{R_{\min }}\left(\frac{M}{m}\right)^{1 / 3} \sqrt{x^{2}+y^{2}+z^{2}}\right|_{\min } \\
\times\left[\frac{(1+\cos f)^{2}}{4}(x \cos f+y \sin f)+\frac{-\sin f \dot{x}+(1+\cos f) \dot{y}}{\sqrt{2}}\right],
\end{gathered}
$$

or by

$$
\begin{aligned}
E_{1}=-E_{2}= & -\left.\frac{G m_{1} m_{2}}{R_{\min }}\left(\frac{M}{m}\right)^{1 / 3} \sqrt{x^{2}+y^{2}+z^{2}}\right|_{\text {min }} \\
& \times\left(\frac{2}{9}\right)^{2 / 3}|t|^{-4 / 3}(x+3 t \dot{x}),
\end{aligned}
$$

in our parabolic and radial formalism, respectively.

Our analytic approach therefore allows us to recast our results in terms of a given minimal distance between the stars (Figures 8 and 9) rather than a given initial separation (Figures 5 and 6). In comparison, the main notable feature is that the scale of Figure 8 is much narrower, i.e., the energy is more sensitive to $R_{\min }$ than to $a$. This is shown more clearly in Figure 7 , where the mean and especially the maximal energy for a fixed $R_{\text {min }}$ are flatter functions of $D$. The maximal energies in units of $\left(G m_{1} m_{2} / R_{\min }\right)(M / m)^{1 / 3}$ are as follows: 1.64 for prograde orbits (obtained for $D=0.12$ ) and 1.60 for retrograde orbits (obtained for $D=0$ ).

\section{EJECTION VELOCITIES}

In the quest for HVSs, the observed quantities are the radial velocity and the star mass. Neglecting the Galactic potential, an ejected $\operatorname{star}\left(E_{1}>0\right)$ with mass $m_{1}$ has a velocity at infinity of $v_{1}=\sqrt{2 E_{1} / m_{1}}$. A comprehensive study of velocity and mass distributions under various assumptions for the binary 


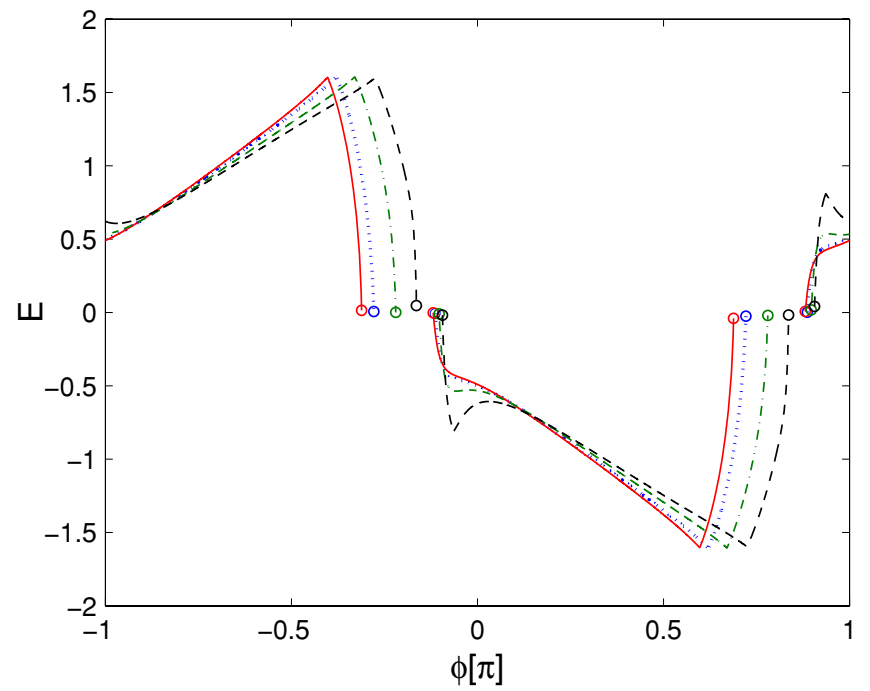

Figure 9. As Figure 6 but for a given minimal distance $R_{\min }$. Energy is in unit of $\left(G m_{1} m_{2} / R_{\min }\right)(M / m)^{1 / 3}$ and the phase in unit of $\pi$.
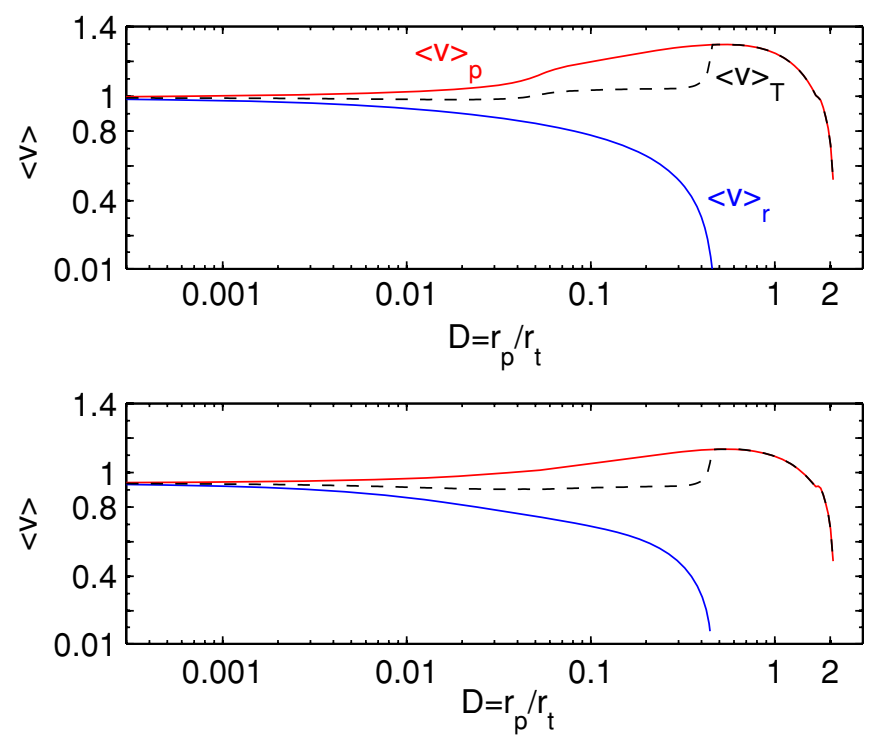

Figure 10. Ejection velocity averaged uniformly over phase as a function of the penetration factor. The average is over 3000 equally spaced $\phi$. Upper panel: the ejection velocity for a given $a$ in units of $\sqrt{2 G m_{2} / a}(M / m)^{1 / 6}$. We plot it for prograde orbits only (red solid line, $\langle v\rangle_{\mathrm{p}}$ ), for retrograde orbits only (blue solid line, $\langle v\rangle_{\mathrm{r}}$ ) and the weighted average over these two orbital directions (black dashed line, $\langle v\rangle_{\mathrm{T}}$ ). See text for details. Lower panel: as upper panel, but for a given minimum possible distance $R_{\min }$ between stars, where $\langle v\rangle$ is in units of $\sqrt{2 G m_{2} / R_{\min }}(M / m)^{1 / 6}$.

population is deferred to a subsequent paper. Here, we calculate the mean velocity for a given binary, assuming that the binary plane is the same as the orbital plane around the $\mathrm{BH}$ and that the binary phase is uniformly distributed. The results are shown in Figure 10 (upper panel) as a function of the penetration factor. If we call $\langle v\rangle_{\mathrm{p}}$ the average velocity for a prograde orbit (red solid line) and $\langle v\rangle_{\mathrm{r}}$ that for a retrograde orbit (blue solid line), then the total average is $\langle v\rangle_{\text {tot }}=n_{\mathrm{p}}\langle v\rangle_{\mathrm{p}}+n_{\mathrm{r}}\langle v\rangle_{\mathrm{r}}$ (blackdashed line), where $n_{\mathrm{r}}$ and $n_{\mathrm{p}}$ are the fractions of ejected stars that originate from retrograde and prograde binaries. Clearly there is no gain in deeper impacts, where the mean saturates at $\sim 0.99 \sqrt{2 G m_{2} / a}(M / m)^{1 / 6}$. Higher velocity-but only by $30 \%$ - can be attained closer to the tidal radius. We also calculate similar means for binaries that, under the $\mathrm{BH}$ tides, shrink to the minimum relative distance $R_{\min }$ (see Section 6). Harder binaries - which would be responsible for the higher mean velocities-would now coalesce instead. This results in an even flatter mean velocity as a function of $D$, which tends to $\sim 0.94 \sqrt{2 G m_{2} / R_{\min }}(M / m)^{1 / 6}$ for $D \rightarrow 0$ (Figure 10 lower panel).

\section{DISCUSSION AND CONCLUSIONS}

The ultimate goal of our work is to statistically characterize the population of stars originating from tidally disrupted binaries and compare it with observations of HVSs.

To this purpose, we derive in this paper the equations of motion and energy for a member of a close binary which suffers an encounter with a third, far more massive body. For the general case, we assume a parabolic orbit for an effective center of mass of the binary (Section 2). This is in contrast with previous works (e.g., Bromley et al. 2006) that considered binaries on hyperbolic or elliptical orbits. However, our assumption is justified since the orbits of binaries that are candidates to produce HVSs are very eccentric. The periapsis passage of a very eccentric orbit could be modeled by a parabolic orbit to a good approximation. This is in agreement with the finding of Bromley et al. (2006) that the initial binary velocity is of little influence on the final outcome. Nevertheless, a binary on a hyperbolic orbit has a total positive energy, and allows for a new disruption channel in which both stars are ejected. This channel is relatively rare, since the energy of the binary is typically small compared to the typical ejection energies. Moreover, such double ejections could not lead to HVSs since the energy is limited to the originalsmall- positive energy of the binary. Our formalism with zero total energy (except the small negative binary binding energy) does not allow for double ejections.

A parabolic trajectory for the binary approaches a radial one for a very close encounter with the massive body. This observation leads us to explicitly adopt a radial orbit with zero energy in order to follow the limiting case of a deep penetrator (Section 3). This simpler set of equations allows us to easily trace a close encounter that otherwise would require high accuracy when calculated with a full three-body code.

Our formalism can be applied quite generally to explore the fate of a binary with arbitrary orbital parameters. However, in this paper, we only focused on results for circular coplanar binaries. The inclination of the binary, as well as its eccentricity, is expected to affect our results quantitatively. We reserve such a study to a forthcoming paper. Nevertheless, we can already reach some conclusions and note quantitative differences with results found in the literature.

The main feature of HVSs is of course their unusually high radial velocity. For equal mass stars, $m_{1}=m_{2}=m_{1,2}$, the expression $G m_{1} m_{2} / R_{\min }$ is simply $m_{1,2} v_{\text {esc }}^{2} / 4$, where $v_{\text {esc }}$ is the escape velocity from the surface of the stars. Since the maximal ejection energy is $1.6 G m_{1} m_{2} / R_{\min }(M / m)^{1 / 3}$ (Figure 7), we derive for the Galactic center $\mathrm{BH}$ a corresponding velocity of $0.9 v_{\text {esc }}(M / m)^{1 / 6} \cong 9 v_{\text {esc }}$. The escape velocity for main sequence stars is about $600-800 \mathrm{~km} \mathrm{~s}^{-1}$ in the mass range of 1-10 $M_{\odot}$. Therefore, velocities can be as large as $\sim 7000 \mathrm{~km} \mathrm{~s}^{-1}$ even for binaries limited to the plane. Scaling for the different masses (and thus $v_{\text {esc }}$ ) assumed by Hills (1988) and by Bromley et al. (2006), we find that a velocity of $0.9 v_{\text {esc }}(M / m)^{1 / 6}$ is respectively a factor of $\sim 1.2$ and $\sim 1.8$ higher than their maximum value of $4000 \mathrm{~km} \mathrm{~s}^{-1}$. Even higher velocities can be achieved when the binary mass ratio is large and the lighter star is ejected. Then, the ejected star travels with maximal velocity 
of $1.3 v_{\text {esc }}(M / m)^{1 / 6}$, which is around $\sim 10,000 \mathrm{~km} \mathrm{~s}^{-1}$ for our Galactic center BH where $M / m \sim 10^{6}$.

For a comparison with observations, it is also important to determine the ejection probability. Bromley et al. (2006) find that the probability for disruption as a function of the penetration factor goes roughly as $P_{\mathrm{ej}} \approx 1-D / 2.2$. The maximum $D$ for disruption is therefore very similar to ours, $D_{\max } \sim 2.1$ (see Figure 10). In contrast, we note a quantitatively different behavior of the probability function for binaries that approach closely the BH, $D \rightarrow 0$. Specifically, their $P_{\mathrm{ej}} \rightarrow 1$ implies that all binaries in this limit are disrupted. This may look intuitively sound since binaries that penetrate so deeply experience very strong tides. However, as we stressed in Section 3, the stars in such binaries separate, but approach each other again on the way back from the BH. In the planar case we find that $20 \%$ of the deeply penetrating binaries survive (Figure 4). Even when taking a uniform distribution for the inclination of the binary plane into account, the percentage of survival is still $10 \%$ for close encounters. In the planar case, Figure 4 indicates that the disruption probability is, in fact, not monotonic in $D$ : for prograde orbits, it peaks at $D=0.15$ and it is almost unity $(98.0-98.8 \%)$ for $0.06<D<0.3$.

For large mass ratios $m_{1} / m_{2}>10$, Bromley et al. (2006) find that the heavier star has consistently more chance to be ejected. We showed here that the probability is $50 \%$. This result is not limited to zero inclination of eccentricity. This fact, together with the rarity of a very massive star, somewhat weakens the claim that the star SO-2 was created by a disruption of a binary in which a $60 M_{\odot}$ companion was ejected (Gould \& Quillen 2003). This conclusion was based on the observed orbital parameters of SO-2. However, the timescale to significantly change its short periapsis distance may quite short, and a careful study of the dynamical processes in the inner regions of the Galactic center is needed to assess it (Perets et al. 2009).

This research was partially supported by an ERC grant, a Packard Fellowship, and a HEFCE PR fellowship. We thank Ehud Nakar and Peter Goldreich for helpful discussions.

\section{REFERENCES}

Antonini, F., Faber, J., Gualandris, A., \& Merritt, D. 2009, arXiv:0909.1959 Bromley, B. C., Kenyon, S. J., Geller, M. J., Barcikowski, E., Brown, W. R., \& Kurtz, M. J. 2006, ApJ, 653, 1194

Brown, W. R., Geller, M. J., Kenyon, S. J., \& Bromley, B. C. 2009, ApJ, 690, L69

Brown, W. R., Geller, M. J., Kenyon, S. J., \& Kurtz, M. J. 2005, ApJ, 622, L33

Brown, W. R., Geller, M. J., Kenyon, S. J., \& Kurtz, M. J. 2006, ApJ, 640, L35

Brown, W. R., Geller, M. J., Kenyon, S. J., Kurtz, M. J., \& Bromley, B. C. 2007, ApJ, 671, 1708

Edelmann, H., Napiwotzki, R., Heber, U., Christlieb, N., \& Reimers, D. 2005, ApJ, 634, L181

Ginsburg, I., \& Loeb, A. 2006a, MNRAS, 368, 221

Ginsburg, I., \& Loeb, A. 2006b, MNRAS, 368, 221

Ginsburg, I., \& Loeb, A. 2007, MNRAS, 376, 492

Gould, A., \& Quillen, A. C. 2003, ApJ, 592, 935

Gualandris, A., Portegies Zwart, S., \& Sipior, M. S. 2005, MNRAS, 363, 223

Hill, G. W. 1886, Acta Math, 8, 1

Hills, J. G. 1988, Nature, 331, 687

Hirsch, H. A., Heber, U., O’Toole, S. J., \& Bresolin, F. 2005, A\&A, 444, L61

Kenyon, S. J., Bromley, B. C., Geller, M. J., \& Brown, W. R. 2008, ApJ, 680, 312

Perets, H. B., Gualandris, A., Kupi, G., Merritt, D., \& Alexander, T. 2009, ApJ, 702, 884

Perets, H. B., Hopman, C., \& Alexander, T. 2007, ApJ, 656, 709

Sesana, A., Haardt, F., \& Madau, P. 2007, MNRAS, 379, L45

Tutukov, A. V., \& Fedorova, A. V. 2009, Astron. Rep., 53, 839

Yu, Q., \& Tremaine, S. 2003, ApJ, 599, 1129 